\title{
Distance function design and Lyapunov techniques for the stability of hybrid trajectories
}

\author{
J. J. Benjamin Biemond ${ }^{\text {a }}$, W. P. Maurice H. Heemels ${ }^{\text {b }}$, Ricardo G. Sanfelice ${ }^{\text {c }}$, \\ Nathan van de Wouw ${ }^{\text {b,d,e }}$

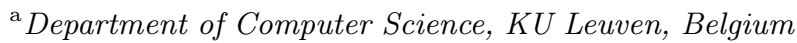 \\ ${ }^{\mathrm{b}}$ Department of Mechanical Engineering, Eindhoven University of Technology, the Netherlands \\ ${ }^{\mathrm{c}}$ Department of Computer Engineering, University of California Santa Cruz, California, U.S.A. \\ ${ }^{\mathrm{d}}$ Department of Civil, Environmental $\&$ Geo- Engineering, University of Minnesota, Pillsbury Drive SE, U.S.A. \\ ${ }^{\mathrm{e}}$ Delft Center for Systems and Control, Delft University of Technology, the Netherlands
}

\begin{abstract}
The comparison between time-varying hybrid trajectories is crucial for tracking, observer design and synchronisation problems for hybrid systems with state-triggered jumps. In this paper, a generic distance function is designed that can be used for this purpose. The so-called "peaking phenomenon", which occurs when using the Euclidean distance to compare two hybrid trajectories, is circumvented by taking the hybrid nature of the system explicitly into account. Based on the proposed distance function, we define the stability of a trajectory and present sufficient Lyapunov-type conditions for hybrid system with statetriggered jumps. A constructive Lyapunov function design is presented for hybrid systems with affine flow and jump maps and a jump set that is a hyperplane. The stability conditions can then be verified using linear matrix conditions. Finally, for this class of systems, we present a tracking controller that asymptotically stabilises a given hybrid reference trajectory and we illustrate our results with an example.
\end{abstract}

Key words:

Hybrid systems; stability analysis; Lyapunov stability; tracking control

\section{Introduction}

Hybrid system models have proven valuable to capture the dynamics of complex systems arising in engineering, biological, and economical systems as these models combine continuous-time dynamics with discrete events or jumps $[11,12]$. While the stability of isolated points or closed sets of hybrid systems is relatively wellunderstood $[11,12]$, the stability of time-varying trajectories received significantly less attention and many issues are presently unsolved. Given the importance

\footnotetext{
* Corresponding author J. J. B. Biemond. Tel. +32 163 27835. Fax +32 16327996 .

Email addresses: benjamin.biemond@cs.kuleuven.be (J. J. Benjamin Biemond), w.p.m.h.heemels@tue.nl (W. P. Maurice H. Heemels), ricardo@ucsc. edu (Ricardo G. Sanfelice), n.v.d.wouw@tue.nl (Nathan van de Wouw).
}

of stability of trajectories in tracking control, observer design and synchronisation problems, it is important to address these open issues.

One of the main complications to study the stability of hybrid trajectories is the "peaking phenomenon" of the Euclidean distance between two trajectories, that can be observed when jump times do not coincide, and the states of two hybrid trajectories are compared at the same continuous-time instant, cf. [4, 15, 18, 22]. Focussing on mechanical systems with unilateral position constraints, the 'peaking phenomenon' has motivated the Zhuravlev-Ivanov method, cf. [6] and related method of [9], in which tracking control and observer problems are defined by requiring the asymptotic stability of a set that consists of the real system and 'mirrored' images. For impacting mechanical systems, in $[10,18,19]$, the standard Euclidean state error is employed away from the impacts times, while near impacts, only the position error, and no velocity error is considered. Alternat- 
ively, measures on complete trajectories are presented in $[7,11]$.

To effectively address stability problems for a large class of hybrid systems, we aim to express stability in terms of a distance function evaluated along trajectories. In [4], this is facilitated by a distance function that takes the jumping nature of the hybrid system into account, therewith avoiding the "peaking phenomenon". For this purpose, a distance function between two states is used which is zero if either both states are equal, or they can become identical after imminent jumps. We note that this implies that the functions considered do not satisfy the conditions to be a metric. However, no constructive design for this distance function was presented in [4]. Focussing on a class of constrained mechanical systems, a similar distance function was employed in [23] to study continuity of trajectories with respect to initial conditions. In both works, ad-hoc techniques were used to design the distance function.

As a first contribution in the current paper, we present a constructive and general design for the distance function. We show that when (global) asymptotic stability is defined with respect to the new distance function, then the proposed distance function provides an intuitively correct comparison between two hybrid trajectories. Subsequently, sufficient conditions for asymptotic stability are presented that rely on Lyapunov functions that may increase during either flow or jump, as long as the Lyapunov function eventually decreases along solutions. For this purpose, maximal or minimal average dwell-time arguments are employed, as proposed in the context of impulsive systems in [13]. The final contribution consists of the application of the developed stability theory to tracking control problems for a class of hybrid systems where the jump map is an affine function of the state, the jump set is a hyperplane, and the continuous-time dynamics can be influenced by a bounded control input. A piecewise affine tracking control law is designed that achieves asymptotic tracking in the proposed distance measure. Finally, the results of this paper are illustrated with an example. Preliminary results have been advertised in [3].

This paper is outlined as follows. We present the class of hybrid systems considered in Section 2. By presenting the constructive distance function design, in Section 3, stability of trajectories is defined and a Lyapunov theorem is formulated. A constructive piecewise quadratic Lyapunov function is designed in Section 4 for a class of hybrid systems with affine jump maps and the jump set contained in a hyperplane. These results are applied to tracking control problems in Section 5. Finally, an example is given in Section 6, followed by conclusions in Section 7.

Notation: Let $\mathbb{N}$ and $\mathbb{N}_{>0}$ denote the set of nonnegative and positive integers, respectively. For a set $X \subset \mathbb{R}^{n}, \partial X$ denotes its boundary and for each $y \in \mathbb{R}^{n}$, the distance between $y$ and $X$ is $\operatorname{dist}(y, X):=\inf _{x \in X}\|x-y\|$. The set $\mathbb{B} \subset \mathbb{R}^{n}$ is the closed unit ball. Given $x \in \mathbb{R}^{n}, y \in \mathbb{R}^{m}$, let $(x, y)$ denote $\left(x^{T}, y^{T}\right)^{T}$. Given a (possibly set-valued) map $F$ with domain of definition $\operatorname{dom} F \subseteq \mathbb{R}^{n}$ and a set $S \subseteq \operatorname{dom} F, F(S)=\{y \mid y \in F(x)$, with $x \in S\}$ denotes its image; $F(y)=\emptyset$ for $y \notin \operatorname{dom} F, F^{k}(x)$, with $x \in \mathbb{R}^{n}, k \in \mathbb{N}_{>0}$, denotes $F\left(F^{k-1}(x)\right)$ and for all $x \in \mathbb{R}^{n}, F^{0}(x)=\{x\}$. We denote the pre-image as $F^{-1}(S)=\{x \mid F(x) \cap S \neq \emptyset\}$. A set-valued map $F: S \subset \mathbb{R}^{n} \rightrightarrows \mathbb{R}^{n}$ is outer semicontinuous if its graph $\left\{(x, y) \in \mathbb{R}^{n} \times \mathbb{R}^{n} \mid x \in S, y \in F(x)\right\}$ is closed, and locally bounded if, for each compact set $\tilde{S} \subseteq S, F(\tilde{S})$ is bounded. For $n, m \in \mathbb{N}_{>0}$, let $I_{n}$ and $O_{m n}$ denote the identity matrix and the matrix of zeros of dimension $n \times n$ and $m \times n$, respectively. Given matrices $A, B \in \mathbb{R}^{n \times n}, A \prec 0$ and $A \preceq 0$ denote that $A$ is symmetric and negative definite or negative semidefinite, respectively.

\section{$2 \quad$ Hybrid system model}

Consider the hybrid system

$$
\begin{aligned}
\dot{x} \in F(t, x) & x \in C, \\
x^{+} \in G(x) & x \in D,
\end{aligned}
$$

with $F:\left[t_{0}, \infty\right) \times C \rightrightarrows \mathbb{R}^{n}$ and $G: D \rightrightarrows \mathbb{R}^{n}$, where $C \subseteq \mathbb{R}^{n}$ and $D \subseteq \mathbb{R}^{n}$. We emphasize that the jump map $G$ is independent of the time $t$, which, in the following, will be exploited in the design of the distance function. In contrast to embedding an extra variable with dynamics $\dot{t}=1$, we prefer to use explicit time-dependency of the flow map $F$, as this allows to study the perturbation of initial conditions without perturbing the initial time. The class of hybrid systems in the form (1) is quite general and permits to model systems arising in many relevant applications, including mechanical systems with impacts [11] and event-triggered control systems, see e.g. [20]. We consider systems (1) that satisfy the following "hybrid basic conditions" (adapted to allow for non-automomous flow maps).

Assumption 1 The data of the hybrid system satisfies

- $C, D$ are closed subsets of $\mathbb{R}^{n}$ with $C \cup D \neq \emptyset$;

- the set-valued mapping $F(t, x)$ is non-empty for all $(t, x) \in\left[t_{0}, \infty\right) \times C$, measurable, and for each bounded closed set $S \subset\left[t_{0}, \infty\right) \times C$, there exists an almost everywhere finite function $m(t)$ such that $\|f\| \leq m(t)$ holds for all $f \in F(t, x)$ and for almost all $(t, x) \in S$;

- $G: D \rightrightarrows \mathbb{R}^{n}$ is nonempty, outer semicontinuous and locally bounded.

We consider solutions $\varphi$ to (1) defined on a hybrid time domain dom $\varphi \subset\left[t_{0}, \infty\right) \times \mathbb{N}$ as given in [11]. The function $\varphi: \operatorname{dom} \varphi \mapsto \mathbb{R}^{n}$ is a solution of (1) when jumps satisfy (1b) and, for fixed $j \in \mathbb{N}$, the function $t \mapsto \varphi(t, j)$ is locally absolutely continuous in $t$ and a Krasovskii solution to (1a). This means $\varphi(t, j) \in D$ and $\varphi(t, j+$ 1) $\in G(\varphi(t, j))$ for all $(t, j) \in \operatorname{dom} \varphi$ such that $(t, j+$ 
1) $\in \operatorname{dom} \varphi$ and $\varphi(t, j) \in C, \frac{d}{d t} \varphi(t, j) \in \bar{F}(t, \varphi(t, j))$ for almost all $t \in I_{j}:=\{t \mid(t, j) \in \operatorname{dom} \varphi\}$ and all $j$ such that $I_{j}$ has nonempty interior. Herein, $\bar{F}(t, x)=$ $\bigcap_{\delta \geq 0} \operatorname{co}\{F(t,(x+\delta \mathbb{B}) \cap C)\}$ and co denotes the closed convex hull operation. We note that this convexification renders $\bar{F}(t, x)$, when restricted to a bounded closed set $S$, convex, outer semi-continuous and measurable in $t$, such that solutions to the differential equation can be defined, cf. [8, Theorem 6, p. 86]. The solution $\varphi$ is said to be maximal if it cannot be extended, complete if $\operatorname{dom} \varphi$ is unbounded, and $\operatorname{dom} \varphi$ is called unbounded in $t$-direction when for each $T \geq t_{0}$ there exist a $j$ such that $(T, j) \in \operatorname{dom} \varphi$.

\section{Design of distance function and stability no- tion}

We restrict our attention to hybrid systems satisfying the following assumption.

Assumption 2 The data of the hybrid system (1) is such that $G$ is a proper function (cf. Definition 1.4.11 in [2]), there is a $k>0$ for which $G^{k}(D) \cap D=\emptyset$ and every maximal solution of (1) has a hybrid time domain that is unbounded in t-direction.

This assumption implies that neither Zeno behaviour nor finite-time escape of solutions are possible.

Definition 1 Consider the hybrid system (1) satisfying Assumption 1 and let $\bar{k}>0$ denote the minimum integer for which Assumption 2 holds. Let the distance function $d:(C \cup D)^{2} \mapsto \mathbb{R}_{\geq 0}$ be defined by

$$
d(x, y)=\inf _{z \in \mathcal{A}}\|(x, y)-z\|
$$

with

$$
\begin{array}{r}
\mathcal{A}:=\left\{\left(z_{x}, z_{y}\right) \in(C \cup D)^{2} \mid \exists k_{1}, k_{2} \in\{0,1, \ldots, \bar{k}\},\right. \\
\left.G^{k_{1}}\left(z_{x}\right) \cap G^{k_{2}}\left(z_{y}\right) \neq \emptyset\right\} .
\end{array}
$$

The following theorem summarises particular properties of the distance function $d$.

Theorem 1 Consider the hybrid system (1) satisfying Assumption 1 and let $\bar{k}$ denote the minimum integer for which Assumption 2 holds. The set $\mathcal{A}$ in (3) is closed and the function d in Definition 1 is continuous and satisfies

1) $d(x, y)=0$ if and only if there exist $k_{1}, k_{2} \in$ $\{0,1, \ldots, \bar{k}\}$ such that $G^{k_{1}}(x) \cap G^{k_{2}}(y) \neq \emptyset$,

2) $\{y \in C \cup D \mid d(x, y)<\beta\}$ is bounded for all $x \in C \cup D$, and all $\beta>0$, and

3) $d(x, y)=d(y, x)$, for all $x, y \in C \cup D$.
PROOF. In order to prove 1), we prove that the infimum in (2) is always attained. First, we observe from Assumption 1 that $G$ is outer semicontinuous, which directly implies that $G^{-1}$ is outer semicontinuous. In addition, as $G$ is proper according to Assumption 2, we observe that $G^{-1}$ is locally bounded, cf. [2].

Since the composition $M_{1} \circ M_{2}$ of set-valued mappings $M_{1}$ and $M_{2}$ is outer semicontinuous and locally bounded when $M_{1}$ and $M_{2}$ are outer semicontinuous and locally bounded, we observe that $G^{k_{2}}$ is outer semicontinuous and locally bounded for all $k_{2} \in\{0,1, \ldots, \bar{k}\}$. In addition, reusing this argument, $G^{-k_{1}} G^{k_{2}}$ is outer semicontinuous and locally bounded for all $k_{1}, k_{2} \in\{0,1, \ldots, \bar{k}\}$. Note that $\mathcal{A}=\cup_{k_{1}, k_{2} \in\{0,1, \ldots, \bar{k}\}} A_{k_{1} k_{2}}$, with $A_{k_{1} k_{2}}:=$ $\left.\left\{(x, y) \in(C \cup D)^{2}\right\rfloor y \in G^{-k_{1}} G^{k_{2}}(x)\right\}$, cf. (3). As, for all $k_{1}, k_{2} \in\{0,1, \ldots, k\}, G^{-k_{1}} G^{k_{2}}$ is outer semicontinuous and locally bounded, and $(C \cup D)^{2}$ is closed, we conclude that each set $A_{k_{1} k_{2}}$ is closed. Consequently, we find that the functions $d_{k_{1} k_{2}}(x, y):=\operatorname{dist}\left((x, y), A_{k_{1} k_{2}}\right)$, for each $k_{1}, k_{2} \in\{0,1, \ldots, \vec{k}\}$, are either continuous functions, or, when $A_{k_{1} k_{2}}=\emptyset$, identical to infinity. Since $A_{00}$ is nonempty, we observe that $d_{00}(x, y)$ is a continuous and locally bounded function in $C \cup D$. We may write $d(x, y)=\min _{k_{1}, k_{2} \in\{0,1, \ldots, \bar{k}\}} d_{k_{1} k_{2}}(x, y)$, proving that $d$ is continuous. As each set $A_{k_{1} k_{2}}$ is closed, $\mathcal{A}$ is closed, such that $d(x, y)=0$ if and only if $(x, y) \in \mathcal{A}$, proving 1$)$.

We now prove 2) by showing that, for every $x \in C \cup D$,

$$
\begin{aligned}
Y_{\infty}(x):=\left\{y \in C \cup D \mid \exists\left(z_{x}, z_{y}\right) \in \mathcal{A},\right. \\
\left.\left\|x-z_{x}\right\| \leq \beta,\left\|y-z_{y}\right\| \leq \beta\right\}
\end{aligned}
$$

is bounded for $\beta>0$. For any $x$, the set $X_{\beta}^{0}:=$ $\left\{w_{x} \mid\left\|w_{x}-x\right\| \leq \beta\right\}$ is compact. Since we have shown above that $G^{-k_{1}} G^{k_{2}}$ is outer semicontinuous and locally bounded for all $k_{1}, k_{2} \in\{0,1, \ldots, \bar{k}\}$, we find that the set $G^{-k_{1}} G^{k_{2}}\left(X_{\beta}^{0}\right)$ is compact for all $k_{1}, k_{2} \in\{0,1, \ldots, \bar{k}\}$. As $z_{y}$ in (4) has to satisfy $z_{y} \in G^{-k_{2}} G^{k_{1}}\left(X_{\beta}^{0}\right)$ for some $k_{1}, k_{2} \in\{0,1, \ldots, \bar{k}\}$, we have shown that $z_{y}$ is contained in a bounded set. Hence, we observe that $Y_{\infty}(x)$ is bounded, which implies 2). Property 3 ) directly follows from symmetry of (3), which completes the proof.

Remark 1 Note that the function d in (2) is not a metric, as it does not satisfy the triangle inequality. Namely, if $G$ is set-valued and, for some $x, G(x)$ contains two distinct points $y$ and $z$, then $d(x, y)=0$ and $d(x, z)=0$ by Definition 1, while $d(y, z) \neq 0$ may still hold in many cases.

An alternative distance function design is presented in Appendix A, which has the advantage that, evaluated along solutions, it yields a continuous function in time. We prefer (2) due to its more simple formulation.

In order to enable the comparison of the states of two trajectories in terms of the distance $d$, similar to [4], we introduce the extended hybrid system with state $q=$ 
$(x, y) \in(C \cup D)^{2}$, flow map

$$
\dot{q} \in F_{e}(t, q):=(F(t, x), F(t, y)),
$$

for $(x, y) \in C_{e}:=C^{2}$ and jumps characterised by

$$
\begin{aligned}
& q^{+}=G_{e}(q):=\left\{\begin{array}{l}
(G(x), y) \text { if } x \in D, y \in C \backslash D \\
(x, G(y)) \text { if } x \in C \backslash D, y \in D \\
\{(G(x), y),(x, G(y))\} \text { if } x, y \in D
\end{array}\right. \\
& \text { for } q \in D_{e}:=\left\{(x, y) \in(C \cup D)^{2} \mid x \in D \vee y \in D\right\}
\end{aligned}
$$

and select the initial condition $\left(\varphi_{x}\left(t_{0}, 0\right), \varphi_{y}\left(t_{0}, 0\right)\right)=$ $\varphi_{q}\left(t_{0}, 0\right)$. We note that the set-valued function $G_{e}$ above motivated the design of the set $\mathcal{A}$ in (3), cf. [4]. Namely, $\mathcal{A}$ represents the smallest set that contains all points $(x, y)$ with $x=y$ that can be forward invariant under $(5)$.

Solutions of this extended system generate a combined hybrid time domain. Introducing $\bar{\varphi}_{x}(t, j):=$ $\left(\begin{array}{ll}I_{n} & O_{n n}\end{array}\right) \varphi_{q}(t, j)$, and $\bar{\varphi}_{y}(t, j):=\left(O_{n n} I_{n}\right) \varphi_{q}(t, j)$, hence allows to evaluate the distance $d\left(\bar{\varphi}_{x}(t, j), \bar{\varphi}_{y}(t, j)\right)$ at every time instant $(t, j) \in \operatorname{dom} \varphi_{q}$.

Given a trajectory $\varphi_{x}$ of (1), we say that a trajectory $\left(\bar{\varphi}_{x}, \bar{\varphi}_{y}\right)$ of $(5)$ represents $\varphi_{x}$ in the first $n$ states when $\bar{\varphi}_{x}$ is a reparameterisation of $\varphi_{x}$. Clearly, any trajectory to (5) represents $\varphi_{x}$ in the first $n$ states when both $\bar{\varphi}_{x}\left(t_{0}, 0\right)=\varphi_{x}\left(t_{0}, 0\right)$ holds and from this initial condition system (1) has a unique solution, as considered in [4].

Definition 2 Consider a hybrid system (1) satisfying Assumption 2 and let $d$ be given in (2). The trajectory $\varphi_{x}$ of (1) is called stable with respect to $d$ if for all $\epsilon>0$ there exists a $\delta(\epsilon)>0$ such that for every initial condition $\varphi_{y}\left(t_{0}, 0\right)$ satisfying $d\left(\varphi_{x}\left(t_{0}, 0\right), \varphi_{y}\left(t_{0}, 0\right)\right) \leq \delta(\epsilon)$, it holds that

$$
d\left(\bar{\varphi}_{x}(t, j), \bar{\varphi}_{y}(t, j)\right)<\epsilon \text { for all }(t, j) \in \operatorname{dom} \varphi_{q}
$$

with $\varphi_{q}(t, j)=\left(\bar{\varphi}_{x}(t, j), \bar{\varphi}_{y}(t, j)\right)$ being any maximal solution to (5) with initial condition $\left(\varphi_{x}\left(t_{0}, 0\right), \varphi_{y}\left(t_{0}, 0\right)\right)$ that represents $\varphi_{x}$ in the first $n$ states, and is called asymptotically stable with respect to $d$ if $\delta$ can be selected such that, in addition,

$$
\lim _{t+j \rightarrow \infty} d\left(\bar{\varphi}_{x}(t, j), \bar{\varphi}_{y}(t, j)\right)=0 .
$$

When the trajectory $\varphi_{x}$ is asymptotically stable with respect to $d$ and (7) holds for all maximal solutions $\varphi_{q}$ to (5), then the trajectory $\varphi_{x}$ is called globally asymptotically stable with respect to $d$.

Remark 2 This stability notion is more general than stability of the set $\mathcal{A}$ in (3) for system (5), since initial conditions of $\varphi_{q}$ in (5) are restricted to $\bar{\varphi}_{x}\left(t_{0}, 0\right)=$ $\varphi_{x}\left(t_{0}, 0\right)$.
To analyse stability using Lyapunov functions that may increase during flow and decrease during jumps, or vice versa, minimal and maximal average inter-jump time are considered as follows.

Definition 3 ([13]) A hybrid time domain $E$ is said to have minimal average inter-jump time $\tau>0$ if there exists $N_{0}>0$ such that for all $(t, j) \in E$ and all $(T, J) \in E$ where $T+J \geq t+j$, it holds that $J-j \leq N_{0}+\frac{T-t}{\tau}$. A hybrid time domain $E$ is said to have maximal average inter-jump time $\tau>0$, if there exists $N_{0}>0$ such that for all $(t, j) \in E$ and all $(T, J) \in E$ where $T+J \geq t+j$, it holds that $J-j \geq \frac{T-t}{\tau}-N_{0}$.

We say that a hybrid trajectory $\varphi_{q}$ has a minimal or maximal average inter-jump time if dom $\varphi_{q}$ has a minimal or maximal average inter-jump time, respectively.

The following theorem presents Lyapunov-based sufficient conditions for the stability of a trajectory $\varphi_{x}$ of (1). As we are interested in stability for given $\varphi_{x}$, these conditions are imposed only near this trajectory.

Theorem 2 Consider a hybrid system (1) satisfying Assumptions 1 and 2. Let d be given in (2). The trajectory $\varphi_{x}$ of system (1) is asymptotically stable with respect to $d$ if there exist a continuous function $V: \mathbb{R}^{n} \times \mathbb{R}^{n} \mapsto \mathbb{R}_{\geq 0}$, $\mathcal{K}_{\infty}$-functions $\alpha_{1}, \alpha_{2}$, a scalar $v_{L}>0$ and scalars $\lambda_{c}, \lambda_{d}$ such that $V$ is continuously differentiable on an open domain containing $V_{L}:=V^{-1}\left(\left[0, v_{L}\right]\right)$ and, for all $(t, j) \in$ dom $\varphi_{x}$, it holds that

$$
\begin{aligned}
& \alpha_{1}\left(d\left(\varphi_{x}(t, j), y\right)\right) \leq V\left(\varphi_{x}(t, j), y\right) \leq \alpha_{2}\left(d\left(\varphi_{x}(t, j), y\right)\right), \\
& \quad \text { for all } y \text { such that }\left(\varphi_{x}(t, j), y\right) \in C_{e} \cup D_{e}, \\
& V(g) \leq e^{\lambda_{d}} V(q), \text { for all } g \in G_{e}(q), \\
& \quad \text { and all } y \text { such that } q=\left(\varphi_{x}(t, j), y\right) \in D_{e} \cap V_{L}, \quad(9) \\
& \left\langle\left.\frac{\partial V}{\partial q}\right|_{q}, f\right\rangle \leq \lambda_{c} V\left(\varphi_{x}(t, j), y\right) \text { for all } f \in \bar{F}_{e}(t, q) \\
& \quad \text { and all } y \text { such that } q=\left(\varphi_{x}(t, j), y\right) \in C_{e} \cap V_{L}, \text { (10) }
\end{aligned}
$$

and at least one of the following conditions are satisfied:

1) $\lambda_{c}<0, \lambda_{d} \leq 0$

2) all trajectories of (1) have minimal average interjump time $2 \tau>0, \lambda_{c} \leq 0$ and $\lambda_{d}+\lambda_{c} \tau<0$;

3) all trajectories of (1) have maximal average interjump time $2 \tau>0, \lambda_{d} \leq 0$ and $\lambda_{d}+\lambda_{c} \tau<0$.

When, in addition, (9) and (10) hold for all y such that $q=\left(\varphi_{x}(t, j), y\right) \in D_{e}$ and $C_{e}$, respectively, then $\varphi_{x}$ is globally asymptotically stable with respect to $d$.

PROOF. The proof is given in Appendix B.

Remark 3 The dependency of $V$ on the trajectory $\varphi_{x}(t, j)$ implies that $V$ in Theorem 2 takes the role of a (hybrid) time-dependent Lyapunov function 
$v(t, j, y)=V\left(\varphi_{x}(t, j), y\right)$, with $(t, j) \in \operatorname{dom} \varphi_{x}$. In this manner, $v(t, j, y)$ characterises the distance $d\left(y, \varphi_{x}(t, j)\right)$ between $\varphi_{x}$ at $(t, j)$ and $y$.

The conditions (8)-(10) are closely related to the Lyapunov conditions used for incremental stability, see e.g. [1, 21] for ordinary differential equations and [16] for hybrid systems where incremental stability is defined with respect to the Euclidean distance, and [24] where incremental stability with respect to non-Euclidean distance functions is investigated for ordinary differential equations. In fact, if the conditions of Theorem 2 hold for any solution $\varphi_{x}(t, j)$ of $(1)$, then they imply asymptotic stability of the set $\mathcal{A}$ of system (5) and, equivalently, an incremental stability property of (1) with respect to the distance d. However, as mentioned above, those conditions need to be satisfied for all $\varphi_{x}$, which makes them stringent and our result relaxes this by requiring (8)-(10) to hold for each point in the range of $\varphi_{x}$ only. Consequently, the conditions in Theorem 2 are less restrictive than the conditions for stability of the set $\mathcal{A}$ obtained using the results of [11].

In fact, the stability of the trajectory $\varphi_{x}$ considered in Theorem 2 is less restrictive than stability of the set $\mathcal{A}$ for the dynamics (5) (or, equivalently, incremental stability of (1)), since the particular trajectory $\varphi_{x}$ is known.

\section{Constructive Lyapunov function design for hybrid systems with affine jump map}

In this section we present the design conditions for the construction of a piecewise quadratic Lyapunov function that, locally, satisfies the requirements (8) and (9). To be able to write the stability conditions in terms of Linear Matrix Inequalities, we need to focus on a class of "linear" hybrid systems: in particular, having singlevalued, affine and invertible jump maps and jump sets characterised by a hyperplane as follows:

$$
\begin{aligned}
\dot{x} & =f(t, x), & & x \in C, \\
x^{+} & =L x+H, & & x \in D
\end{aligned}
$$

with the function $f$ measurable in its first argument and Lipschitz in its second argument, the matrix $L \in \mathbb{R}^{n \times n}$ being invertible, and $H \in \mathbb{R}^{n}$. Furthermore, the sets $C$ and $D$ are nonempty, closed and satisfy

$$
\begin{aligned}
C \subseteq\left\{x \in \mathbb{R}^{n} \mid J x+K \leq 0 \wedge\right. \\
\left.\quad\left(J L^{-1} x+K-J L^{-1} H\right) s \leq 0\right\}, \\
D:=\left\{x \in C \mid J x+K=0 \wedge z_{1} x+z_{2} \leq 0\right\},
\end{aligned}
$$

where the parameters $J^{T}, z_{1}^{T} \in \mathbb{R}^{n} \backslash\{0\}, K, z_{2} \in \mathbb{R}$ characterise the half hyperplane containing $D$, and $s \in$ $\{-1,1\}$ is selected such that $n_{g d}:=s\left(L^{-1}\right)^{T} J^{T}$ is a normal vector to $G(D)$ pointing out of $C$. Let $G(D) \subset C$ and the following assumption hold.

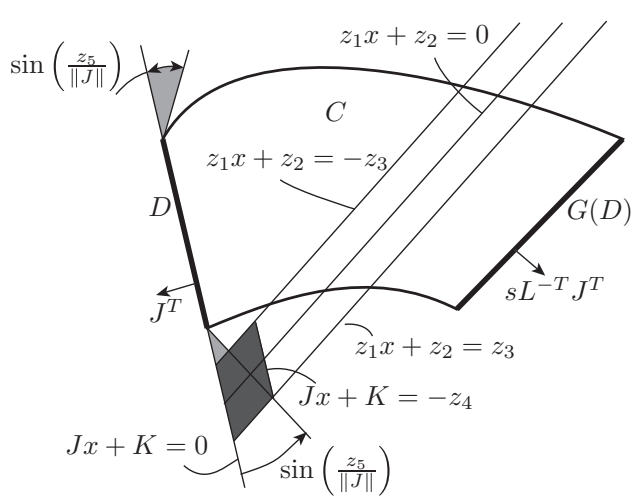

Figure 1. Pictorial illustration of the phase space of (11) when Assumption 3 is satisfied. The second and third bullet of this assumption imply that the intersection between $C$ and the domains depicted in dark gray and light gray, respectively, is empty.

Assumption 3 The data of (11) is such that there exist scalars $z_{3}, z_{4}, z_{5}>0$ such that

- $z_{1} x+z_{2} \geq z_{3}$ for all $x \in G(D)$,

- $J x+K<-z_{4}$ for all $x \in C$ that satisfy $\left|z_{1} x+z_{2}\right| \leq z_{3}$,

- for all $x \in C$ with $z_{1} x+z_{2} \leq 0$, there exists a $y \in D$ such that $J x+K \leq-z_{5}\|x-y\|$,

- all maximal solutions of (11) are complete.

The first three bullets of this assumption are illustrated in Fig. 1. Note that this assumption directly implies $D \cap G(D)=\emptyset$, cf. Assumption 2. All solutions to (11) have a time domain that is unbounded in $t$-direction, as, firstly, $G(D) \cap D=\emptyset$ excludes Zeno-behaviour since $D$ is closed, secondly, $G$ is linear and, thirdly, $f$ is Lipschitz in its second argument. Hence, Assumption 3 implies that Assumptions 1 and 2 hold for system (11). In Section 6 , we present an example of a mechanical system that satisfies (11) and Assumption 3.

In order to present a constructive Lyapunov function design, we first introduce the function $\bar{G}: \mathbb{R}^{n} \mapsto \mathbb{R}^{n}$ as

$$
\bar{G}(x):=L x+H+M(J x+K)+s L J^{T} \max \left(0, z_{1} x+z_{2}\right),
$$

where the parameter $M \in \mathbb{R}^{n}$ is to be designed. Note that if $x \in D$, then $\bar{G}(x)=G(x)=L x+H$.

Since $G(D) \cap D=\emptyset$, Definition 1 implies that $d(x, y)=0$ if and only if $x=y$, or $x=G(y)$, or $y=G(x)$. To design a Lyapunov function $V$, we note that (8) requires that $V(x, y)=0$ if and only if $d(x, y)=0$. Hence, we propose the following piecewise quadratic Lyapunov function:

$V(x, y)=\min \left(\|x-y\|_{P_{0}}^{2},\|x-\bar{G}(y)\|_{P_{s}}^{2},\|\bar{G}(x)-y\|_{P_{s}}^{2}\right)$,

where the positive definite matrices $P_{0}, P_{s} \in \mathbb{R}^{n \times n}$ are to be designed. While this function is not smooth, we restrict our attention to a sufficiently small sub-level set where, as we will show in Lemma 3, the function $V$ is smooth. 


\section{Design of Lyapunov function parameters}

To design the parameters $P_{0}, P_{s}$ and $M$ of the Lyapunov function $V$ in (13), we employ the following lemma.

Lemma 3 Consider the hybrid system (11), let $M \in$ $\mathbb{R}^{n}$ satisfy $\left(J L^{-1} M+1\right) s<0$, let $P_{0}, P_{s} \succ 0$ and let Assumption 3 hold. Consider the function $V$ in (13). If for some $\lambda_{d} \in \mathbb{R}$ it holds that

$$
\begin{aligned}
(L+M J)^{T} P_{s}(L+M J) & \preceq e^{\lambda_{d}} P_{0}, \\
P_{0} & \preceq e^{\lambda_{d}} P_{s},
\end{aligned}
$$

then there exist $\mathcal{K}_{\infty}$-functions $\alpha_{1}, \alpha_{2}$ and $v_{L}>0$ such that the conditions (8) and (9) in Theorem 2 are satisfied with $V_{L}=V^{-1}\left(\left[0, v_{L}\right]\right)$ and the function $V$ in $(13)$ is smooth on an open domain containg $V_{L}$.

PROOF. The proof is given in Appendix B.

This lemma provides sufficient conditions on the hybrid systems (11) and the Lyapunov function (13) such that the conditions (8) and (9) are satisfied. In the following section, we present a tracking control law, and additional conditions on $V$, such that the other conditions in Theorem 2 are also satisfied.

\section{Tracking control problems}

We now employ the results on the asymptotic stability of jumping hybrid trajectories to solve a tracking problem of a hybrid trajectory with jumps.

We restrict our attention to tracking control problems for the class of systems (11) with $f(t, x)=A x+E+$ $B u(t, x), A \in \mathbb{R}^{n \times n}, E, B \in \mathbb{R}^{n}$, with a control law $u:[0, \infty) \times C \mapsto \mathbb{R}$ to be designed. In the scope of this tracking problem, we consider a reference trajectory $x_{d}$, which is a solution to (11) for a feedforward input signal $u(t, x)=u_{\mathrm{ff}}(t)$. We assume that $y$ is a trajectory that is generated by the control signal $u(t, y)=u_{\mathrm{ff}}(t)+u_{\mathrm{fb}}(t, y)$, and assume that $u_{\mathrm{fb}}$ vanishes along the trajectory $x_{d}$, i.e. $u_{\mathrm{fb}}\left(t, x_{d}(t, j)\right)=0$ for almost all $(t, j) \in \operatorname{dom} x_{d}$ (appropriate designs for $u_{\mathrm{fb}}$ will depend on the known trajectory $x_{d}$ ). Hence, the flow map of the extended hybrid system (5) is given by

$$
F_{e}\left(t, x_{d}, y\right)=\left(\begin{array}{c}
A x_{d}+E+B\left(u_{\mathrm{ff}}(t)+u_{\mathrm{fb}}\left(t, x_{d}\right)\right) \\
A y+E+B\left(u_{\mathrm{ff}}(t)+u_{\mathrm{fb}}(t, y)\right)
\end{array}\right)
$$

We partition $C_{e} \cup D_{e}$ in the three sets $S_{0}, S_{1}, S_{2}$ where the minimiser of $(13)$ is $\|x-y\|_{P_{0}}^{2},\|x-\bar{G}(y)\|_{P_{s}}^{2}$ or $\|\bar{G}(x)-y\|_{P_{s}}^{2}$, respectively. Introducing the function $\bar{x}_{d}(t):=x_{d}\left(t, \min _{(t, j) \in \operatorname{dom} x_{d}} j\right)$, we design a switching feedback law $u_{f b}$ as:

$$
u_{\mathrm{fb}}(t, y)=\left\{\begin{array}{c}
-c_{0}\left(\bar{x}_{d}(t)-y\right) \\
\text { for }\left(\bar{x}_{d}(t), y\right) \in S_{0} \\
-\frac{\beta_{2}^{T}}{\beta_{2}^{T} \beta_{2}} \beta_{1}(t)-c_{1}\left(\bar{x}_{d}(t)-\bar{G}(y)\right), \\
\text { for }\left(\bar{x}_{d}(t), y\right) \in S_{1} \\
-\frac{\beta_{4}^{T}}{\beta_{4}^{T} \beta_{4}} \beta_{3}(t)-c_{2}\left(\bar{G}\left(\bar{x}_{d}(t)\right)-y\right), \\
\text { for }\left(\bar{x}_{d}(t), y\right) \in S_{2}
\end{array}\right.
$$

with $c_{0}^{T}, c_{1}^{T}, c_{2}^{T} \in \mathbb{R}^{n}$,

$$
\begin{aligned}
& \beta_{1}(t)=\left(\begin{array}{ll}
I_{n} & -L-M J
\end{array}\right)\left(\begin{array}{c}
A \bar{x}_{d}(t)+B u_{\mathrm{ff}}(t)+E \\
A \bar{G}^{\circ}\left(\bar{x}_{d}(t)\right)+B u_{\mathrm{ff}}(t)+E
\end{array}\right), \\
& \beta_{3}(t)=\left(\begin{array}{ll}
L+M J & -I_{n}
\end{array}\right)\left(\begin{array}{c}
A \bar{x}_{d}(t)+B u_{\mathrm{ff}}(t)+E \\
A \bar{G}\left(\bar{x}_{d}(t)\right)+B u_{\mathrm{ff}}(t)+E
\end{array}\right),
\end{aligned}
$$

$\beta_{2}=-(L+M J) B$ and $\beta_{4}=-B$, where $\bar{G}^{\circ}(x)$ is designed as $\bar{G}^{\circ}(x)=(L+M J)^{-1}(x-H-M K)$, which, restricted to $S_{1} \cap V_{L}$, coincides with the inverse of $\bar{G}$.

Using this switched control law, which switches on the basis of the Lyapunov function designed in (13), we formulate in the following result explicit conditions on the controller parameters $c_{0}, c_{1}, c_{2}, M, P_{0}$ and $P_{s}$ under which the tracking problem is solved.

Theorem 4 Consider the hybrid system (11) with $f(t, x)=A x+E+B\left(u_{\mathrm{ff}}(t)+u_{\mathrm{fb}}(t, x)\right)$, for some measurable function $u_{\mathrm{ff}}(t)$ and let $x_{d}$ be a solution of (11) for $u_{\mathrm{fb}} \equiv 0$. Let $P_{0}, P_{s} \in \mathbb{R}^{n \times n}, M \in \mathbb{R}^{n}$, consider $V$ as in (13) and let $u_{\mathrm{fb}}$ be designed as in (17), with $\bar{x}_{d}(t)=x_{d}\left(t, \min _{(t, j) \in \text { dom } x_{d}} j\right)$ and $c_{0}^{T}, c_{1}^{T}, c_{2}^{T} \in \mathbb{R}^{n}$. Let $L+M J$ be invertible and $B \neq 0$.

Let the assumptions of Lemma 3 hold for $\lambda_{d} \in \mathbb{R}$, let all trajectories of (11) have a time domain that is unbounded in t-direction, and assume

$$
\beta_{1}(t) \in \operatorname{span}\left(\beta_{2}\right), \quad \text { and } \beta_{3}(t) \in \operatorname{span}\left(\beta_{4}\right)
$$

hold for almost all $t$.

Let, for some $\lambda_{c} \in \mathbb{R}$, the following LMIs be satisfied:

$$
\begin{array}{r}
\left(A+B c_{0}\right)^{T} P_{0}+P_{0}\left(A+B c_{0}\right)-\lambda_{c} P_{0} \preceq 0, \\
P_{s}\left(\beta_{2} c_{1}+(L+M J) A(L+M J)^{-1}\right)+\left(\beta_{2} c_{1}+\right. \\
\left.(L+M J) A(L+M J)^{-1}\right)^{T} P_{s}+\lambda_{c} P_{s} \preceq 0, \\
P_{s}\left(A+B c_{2}\right)+\left(A+B c_{2}\right)^{T} P_{s}+\lambda_{c} P_{s} \preceq 0 .
\end{array}
$$

If either of the following cases hold, then the trajectory $x_{d}$ is asymptotically stable with respect to $d$.

1) $\lambda_{c}<0, \lambda_{d} \leq 0$ 
2) all trajectories of (1) have minimal average interjump time $2 \tau>0, \lambda_{c} \leq 0$ and $\lambda_{d}+\lambda_{c} \tau<0$,

3) all trajectories of (1) have maximal average interjump time $2 \tau>0, \lambda_{d} \leq 0$ and $\lambda_{d}+\lambda_{c} \tau<0$.

PROOF. The proof is given in Appendix B.

\section{Example}

We now present hybrid system and design a control law for which a maximal dwell-time argument proves asymptotic stability of the reference trajectory. Consider a single degree-of-freedom system with a damper with damping constant $c>0$ and a spring with stiffness $k>0$ and unloaded position $x=\bar{x}_{1}$, as shown in Fig. 2. Impacts can only occur at the constraint at $x_{1}=0$. Let the

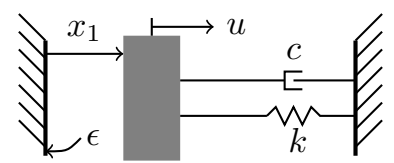

Figure 2. Dissipative mechanical system.

impacts be described by a restitution coefficient $\varepsilon=0.9$. Hence, the impacts are dissipative, which allows to study the stability of the trajectory using a maximal average inter-jump time result. Assuming that finite constraint forces can be ignored, i.e. persistent contact does not occur, the hybrid system is described by (11) with

$A=\left(\begin{array}{cc}0 & 1 \\ -k & -c\end{array}\right), B=\left(\begin{array}{l}0 \\ 1\end{array}\right), E=\left(\begin{array}{c}0 \\ k \bar{x}_{1}\end{array}\right), L=-\varepsilon I_{2}$, $J=\left(\begin{array}{ll}1 & 0\end{array}\right), K=0, H=0, s=-1, z_{1}=\left(\begin{array}{ll}0 & 1\end{array}\right), z_{2}=0$ and the set $C$ is selected to exclude the origin. The parameters $\bar{x}_{1}=1, k=1$ and $c=0.02$ are used.

Let the reference trajectory $x_{d}$ be a solution to (11) for a feedforward function $u=u_{\mathrm{ff}}(t)=100 \cos (\omega t)$, with $\omega=0.4$. This forcing is selected such that the reference trajectory $x_{d}$ with initial condition $x_{d}(0,0)=(50,0)$ has a maximal average inter-jump time $\tau_{d}>0$. In addition, $\left\|x_{d}(t, j)\right\|>s$ for all $(t, j) \in \operatorname{dom} x_{d}$, for some $s>0$, i.e. $x_{d}$ does not tend to the origin.

We now apply the constructive control law design proposed in Section 5 to enforce tracking of the trajectory $x_{d}$. Selecting $P_{0}=\left(\begin{array}{ll}k & 0 \\ 0 & 1\end{array}\right)$ and $P_{s}=\frac{1}{\varepsilon} P_{0}$, we observe that the conditions of Lemma 3 are satisfied with $\lambda_{d}=$ $\log (\varepsilon)<0$. In addition, we observe that $c_{0}=c_{1}=c_{2}=0$ can be selected, such that (19)-(21) hold with $\lambda_{c}=0$, as $P_{0} A+A^{T} P_{0}=\left(\begin{array}{cc}0 & 0 \\ 0 & -2 c\end{array}\right)$ and $P_{s} A+A^{T} P_{s}=\left(\begin{array}{cc}0 & 0 \\ 0 & -\frac{2 c}{\varepsilon}\end{array}\right)$.
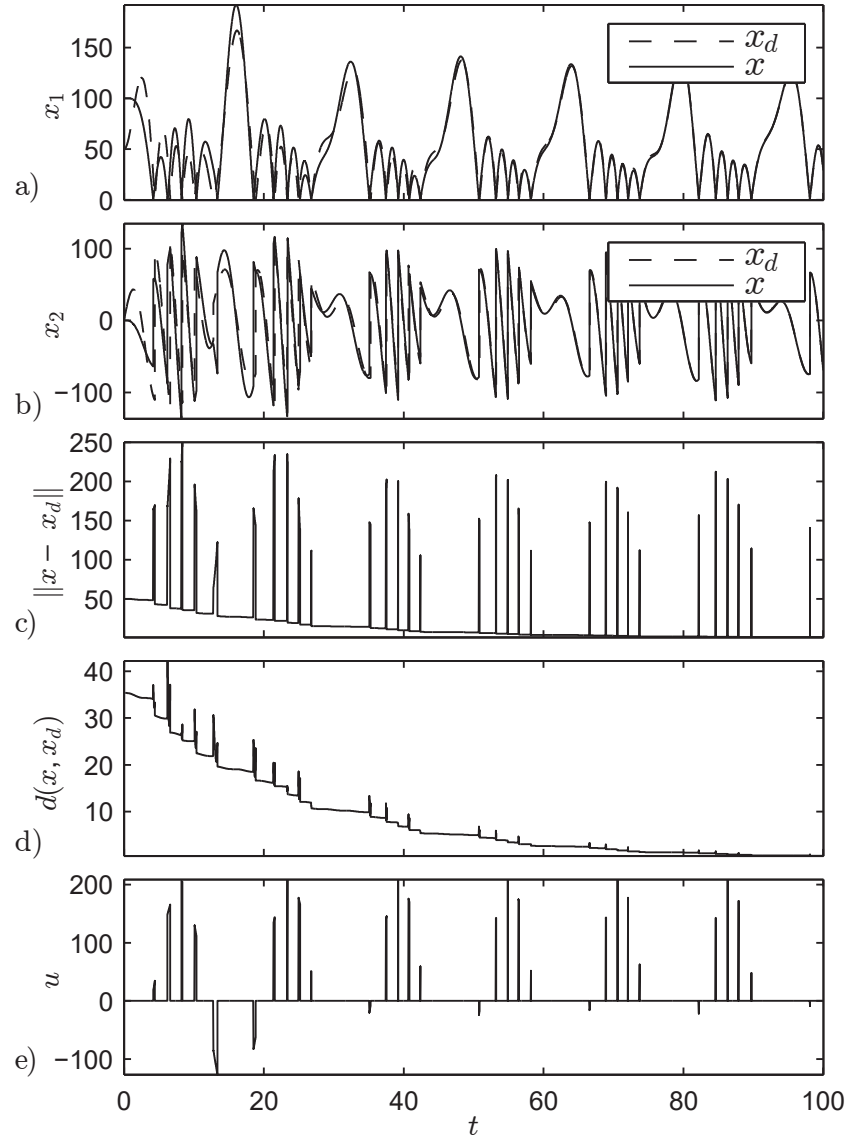

Figure 3. a) and b) Reference trajectory $x_{d}$ and plant trajectory $x$ for the dissipative mechanical system and periodic forcing. c) Euclidean tracking error. d) Distance function (2). e) Control force $u$.

Then, (17) yields the control law:

$$
u_{\mathrm{fb}}(t, y)= \begin{cases}0, & \left(\bar{x}_{d}(t), y\right) \in S_{0} \\ -\frac{1+\varepsilon}{\varepsilon}\left(k \bar{x}_{1}+u_{\mathrm{ff}}(t)\right), & \left(\bar{x}_{d}(t), y\right) \in S_{1} \\ -(1+\varepsilon)\left(k \bar{x}_{1}+u_{\mathrm{ff}}(t)\right), & \left(\bar{x}_{d}(t), y\right) \in S_{2}\end{cases}
$$

As the trajectory $x_{d}$ has a maximal average inter-jump time, denoted $\tau_{d}$, nearby trajectories will have the same behaviour. Hence, selecting $v_{L}>0$ sufficiently small and restricting our attention to the hybrid system (5) with flow set $C_{e} \cap V_{L}$ and jump set $D_{e} \cap V_{L}$, with $V_{L}=$ $V^{-1}\left(\left[0, v_{L}\right]\right)$, we conclude that $x$ also has a maximal average dwell-time $\tau_{x}$, with $\tau_{x}$ close to $\tau_{d}$. Hence, the trajectory of the embedded system (5) has a maximal average inter-jump time $\frac{\max \left(\tau_{d}, \tau_{x}\right)}{2}>0$. Consequently, case 3 ) of Theorem 4 proves that the trajectory is (locally) asymptotically stabilised with respect to $d$ by the control law (22).

In Fig. 3, the performance of this controller is illustrated and a trajectory with initial condition $x(0,0)=(100,0)$ is shown. The achieved stability of $x_{d}$ with respect to $d$ clearly corresponds to desirable behaviour.

From the structure of the control law (22), we observe 
that no control is active when $V\left(\bar{\varphi}_{y}(t, j), x_{d}(t, j)\right)=$ $\left\|\varphi_{y}(t, j)-x_{d}(t, j)\right\|_{P_{0}}^{2}$. In fact, the dissipative effect of both the damping force $c \dot{x}$ and the jump map implies that no control is needed during these time intervals. The control input $u$ only needs to compensate the destabilising effect of the forcing term $E+B u_{\mathrm{ff}}$ during the "peaks" of the Euclidean error.

\section{Conclusion}

In this paper, we considered the stability of time-varying and jumping trajectories of hybrid systems with statetriggered jumps. This requires the comparison of different trajectories of a hybrid system for which we proposed a novel systematic distance function design, because the standard Euclidean distance is not adequate. Sufficient conditions for stability in terms of this distance function were formulated using Lyapunov functions that can exploit maximum or minimum average inter-jump time properties and that have sub-level sets that can be partitioned in disconnected domains. In fact, when the jump map is an affine function and the jump set a hyperplane, a systematic design procedure for piecewise quadratic Lyapunov functions was proposed as well. Based on the general theory and the specific matrix conditions for the piecewise quadratic Lyapunov function design, we designed a switched tracking control law for hybrid systems that only allow control during flow. A numerical example illustrates the applicability of our results leading to a control law that achieves accurate tracking. Moreover, the example nicely shows that the presented distance function and the corresponding asymptotic stability notion do indeed correspond to desired tracking behaviour.

\section{Acknowledgements}

J.J.B. Biemond received support as FWO Pegasus Marie Curie Fellow, from FWO project G071711N and from OPTEC, the Optimization in Engineering Center of KU Leuven. This research is supported partially by the European Union Seventh Framework Programme [FP7/2007-2013] under grant agreement no. 257462 HYCON2 Network of excellence, the National Science Foundation under CAREER Grant no. ECS-1150306 and by the Air Force Office of Scientific Research under YIP Grant no. FA9550-12-1-0366.

\section{References}

[1] D. Angeli. A Lyapunov approach to incremental stability properties. IEEE Transactions on Automatic Control, $47(3): 410-421,2002$.

[2] J.-P. Aubin and H. Frankowska. Set-Valued Analysis. Modern Birkhäuser Classics. Birkhäuser, Boston, 2009.
[3] J. J. B. Biemond, W. P. M. H. Heemels, R. G. Sanfelice, and N. van de Wouw. Constructing distance functions and piecewise quadratic Lyapunov functions for stability of hybrid trajectories. In Proceedings of the 54th IEEE Conference on Decision and Control, December 15-18, 2015, Osaka, Japan, 2015.

[4] J. J. B. Biemond, N. van de Wouw, W. P. M. H. Heemels, and H. Nijmeijer. Tracking control for hybrid systems with statetriggered jumps. IEEE Transactions on Automatic Control, 58(4):876-890, 2013.

[5] J. J. Benjamin Biemond, W. P. Maurice H. Heemels, Ricardo G. Sanfelice, and Nathan van de Wouw. Distance function design and Lyapunov techniques for the stability of hybrid trajectories. Technical report, 2014. Arxiv: https://arxiv.org/abs/1501.00161.

[6] B. Brogliato. Nonsmooth Mechanics. Springer-Verlag, London, 1999.

[7] M. Broucke and A. Arapostathis. Continuous selections of trajectories of hybrid systems. Systems 8 Control Letters, 47:149-157, 2002.

[8] A. F. Filippov. Differential equations with discontinuous righthand sides, volume 18 of Mathematics and its applications (Soviet Series). Kluwer Academic Publishers, Dordrecht, 1988.

[9] F. Forni, A. R. Teel, and L. Zaccarian. Follow the bouncing ball: global results on tracking and state estimation with impacts. IEEE Transactions Automatic Control, 58(6):14701485, 2013.

[10] S. Galeani, L. Menini, A. Potini, and A. Tornambè. Trajectory tracking for a particle in elliptical billiards. International Journal of Control, 81(2):189-213, 2008.

[11] R. Goebel, R. G. Sanfelice, and A. R. Teel. Hybrid dynamical systems: Modeling, Stability and Robustness. Princeton University Press, Princeton, 2012.

[12] W. P. M. H. Heemels, B. de Schutter, J. Lunze, and M. Lazar. Stability analysis and controller synthesis for hybrid dynamical systems. Philosophical Transactions of the Royal Society A: Mathematical, Physical and Engineering Sciences, 368(1930):4937-4960, 2010.

[13] J. P. Hespanha, D. Liberzon, and A. R. Teel. Lyapunov conditions for input-to-state stability of impulsive systems. Automatica, 44(11):2735 - 2744, 2008.

[14] H. K. Khalil. Nonlinear Systems. Prentice Hall, Upper Saddle River, third edition, 2002.

[15] R. I. Leine and N. van de Wouw. Stability and convergence of mechanical systems with unilateral constraints, volume 36 of Lecture Notes in Applied and Computational Mechanics. Springer-Verlag, Berlin, 2008.

[16] Y. Li, S. Phillips, and R. G. Sanfelice. Basic properties and characterizations of incremental stability prioritizing flow time for a class of hybrid systems. Systems $\mathcal{G}$ Control Letters, 90:7-15, 2016.

[17] J. Lygeros, K. H. Johansson, S. N. Simić, Jun Zhang, and S. S. Sastry. Dynamical properties of hybrid automata. IEEE Transactions on Automatic Control, 48(1):2-17, 2003.

[18] L. Menini and A. Tornambè. Asymptotic tracking of periodic trajectories for a simple mechanical system subject to nonsmooth impacts. IEEE Transactions on Automatic Control, 46(7):1122-1126, 2001.

[19] I. C. Morărescu and B. Brogliato. Trajectory tracking control of multiconstraint complementarity Lagrangian systems. IEEE Transactions on Automatic Control, 55(6):1300-1313, 2010 . 
[20] R. Postoyan, P. Tabuada, D. Nesic, and A. Anta. A framework for the event-triggered stabilization of nonlinear systems. IEEE Transactions on Automatic Control, 60(4):982-996, 2015.

[21] B. S. Rüffer, N. van de Wouw, and M. Mueller. Convergent systems vs. incremental stability. Systems $\&$ Control Letters, 62(3):277-285, 2013.

[22] R. G. Sanfelice, J. J. B. Biemond, N. van de Wouw, and W. P. M. H. Heemels. An embedding approach for the design of state-feedback tracking controllers for references with jumps. International Journal of Robust and Nonlinear Control, 24(11):1585-1608, 2014.

[23] M. Schatzman. Uniqueness and continuous dependence on data for one-dimensional impact problems. Mathematical and Computer Modelling, 28(4-8):1-18, 1998.

[24] M. Zamani, N. van de Wouw, and R. Majumdar. Backstepping controller synthesis and characterizations of incremental stability. Systems $\& 5$ Control Letters, 62(10):949$962,2013$.

\section{A Alternative distance function}

The distance function (2) is not necessarily continuous over jumps when evaluated along solutions to (1). When $G$ is a single-valued and invertible function, such a continuity property could be induced by the function:

$$
d_{Q}(x, y)=\inf _{\substack{N \in \mathbb{N} \\
\begin{array}{c}
\left(x^{i}, y^{i}\right) \in \mathcal{A}, i=1, \ldots, N \\
y^{0}=x, x^{N+1}=y
\end{array}}} \sum_{i=0}^{N}\left\|y^{i}-x^{i+1}\right\|,
$$

that coincides with the quotient metric on the quotient space generated by the equivalence $x \sim y$ if $(x, y) \in \mathcal{A}$. This quotient space has been suggested in [17] to study hybrid systems. We note that when $G$ is non-invertible, then $d_{Q}(x, y)=0 \Leftrightarrow(x, y) \in \mathcal{A}$ may not hold. To allow for non-invertible jump maps, we prefer the distance function $d$ in (2) over $d_{Q}$.

\section{B Proofs}

PROOF. [Proof of Theorem 2] We restrict our attention to maximal trajectories $\varphi_{q}$ to $(5)$ that represent $\varphi_{x}$ in the first $n$ states. These trajectories always exist, which follows from the comparison of (1) and (5) and the fact that $\varphi_{x}$ is a trajectory to (1). The observation that $\bar{\varphi}_{y}$ is a reparameterisation of a trajectory $\varphi_{y}$ for (1), and both $\varphi_{x}$ and $\varphi_{y}$ are unbounded in $t$-direction by Assumption 2, proves that the trajectory $\varphi_{q}$ is unbounded in $t$-direction.

We first prove that $V\left(\varphi_{q}(t, j)\right)<v_{L}$ for all $(t, j) \in$ $\operatorname{dom} \varphi_{q}$ and all trajectories $\varphi_{q}$ of $(5)$ if $\bar{k} V\left(\varphi_{q}\left(t_{0}, 0\right)\right)<$ $v_{L}$, where $\bar{k}$ is chosen as $\bar{k}=1$ if 1 ) holds, $\bar{k}=e^{\lambda_{d} N_{0}}$ if 2) holds and $\lambda_{d} \geq 0$, and $\bar{k}=e^{\lambda_{c} N_{0} \tau}$ if 3) holds and $\lambda_{c} \geq 0$, with $N_{0}$ given in Definition 3. Observe that if all trajectories of (1) have a minimal or maximal average inter-jump time $2 \tau$, then (5) has minimal or maximal average interjump time $\tau$.

To prove that the values of $\bar{k}$ defined above are appropriate, for the sake of contradiction, suppose that $\bar{k} V\left(\varphi_{q}\left(t_{0}, 0\right)\right)<v_{L}$ and there exists a time $\left(t_{0}+\bar{T}, \bar{J}\right) \in$ $\operatorname{dom} \varphi_{q}, \bar{T}, \bar{J} \geq 0$, such that $V\left(\varphi_{q}\left(t_{0}+\bar{T}, \bar{J}\right)\right) \geq v_{L}$. Hence, there exist $T \leq \bar{T}$ and $J \leq \bar{J}$ such that $\left(t_{0}+\right.$ $T, J) \in \operatorname{dom} \varphi_{q}$ and

$$
V\left(\varphi_{q}\left(t_{0}+T, J\right)\right) \geq v_{L},
$$

but $V\left(\varphi_{q}(t, j)\right)<v_{L}$ for all $(t, j) \in R:=\{(t, j) \in$ $\left.\operatorname{dom} \varphi_{q} \mid t<t_{0}+T \vee j<J\right\}$.

Since $\varphi_{q}$ represents $\varphi_{x}$ in the first $n$ states, (9)-(10) imply that $V(g) \leq e^{\lambda_{d}} V\left(\varphi_{q}(t, j)\right)$ and $\left\langle\left.\frac{\partial V}{\partial q}\right|_{\varphi_{q}(t, j)}, f\right\rangle \leq$ $\lambda_{c} V\left(\varphi_{q}(t, j)\right)$ hold for all $(t, j) \in R, f \in \bar{F}_{e}\left(t, \varphi_{q}(t, j)\right)$ and $g \in G_{e}\left(\varphi_{q}(t, j)\right)$.

Analogue to [22], we study the function $(t, j) \mapsto$ $w(t, j):=V\left(\bar{\varphi}_{x}(t, j), \bar{\varphi}_{y}(t, j)\right)$ along the given solution $\varphi_{q}$ over the time domain $R$ and we introduce scalars $\left\{t_{j}\right\}$ such that $R=\bigcup_{j}\left(\left[t_{j}, t_{j+1}\right] \times\{j\}\right)$. As, for each $j$, the functions $\bar{\varphi}_{x}, \bar{\varphi}_{y}$ are absolutely continuous in $t$ in the time interval $\left[t_{j}, t_{j+1}\right] \times\{j\}$, $w(t, j)$ is absolutely continuous in $t$ as well. Evaluating $\dot{w}(t, j)=\frac{\partial V}{\partial q} f$ for some $f \in \bar{F}_{e}\left(t,\left(\bar{\varphi}_{x}(t, j), \bar{\varphi}_{y}(t, j)\right)\right)$, we find with (10) that $\dot{w}(t, j) \leq \lambda_{c} w(t, j)$. With the comparison lemma, [14, Lemma 3.4], we find $w\left(t_{j+1}, j\right)=e^{\lambda_{c}\left(t_{j+1}-t_{j}\right)} w\left(t_{j}, j\right)$ for all $j$. For a subsequent jump, (9) yields $w\left(t_{j+1}, j+1\right)=e^{\lambda_{d}} w\left(t_{j+1}, j\right)$. Applying this result repetitively, we find

$w\left(t_{0}+T, j\right)=V\left(\varphi_{q}\left(t_{0}+T, J\right)\right) \leq e^{\lambda_{c} T+\lambda_{d} J} V\left(\varphi_{q}\left(t_{0}, 0\right)\right)$.

If case 1) of the theorem holds, we directly observe $V\left(\varphi_{q}\left(t_{0}+T, J\right)\right) \leq V\left(\varphi_{q}\left(t_{0}, 0\right)\right)$, contradicting (B.1). If $\lambda_{d} \geq 0$ and case 2) holds, then the definition of minimal average inter-jump time yields $\lambda_{c} T+\lambda_{d} J \leq$ $\frac{T}{\tau}\left(\lambda_{c} \tau+\lambda_{d}\right)+\lambda_{d} N_{0} \leq \lambda_{d} N_{0}$, such that with (B.2) we find $V\left(\varphi_{q}\left(t_{0}+T, J\right)\right) \leq \bar{k} V\left(\varphi_{q}\left(t_{0}, 0\right)\right)<v_{L}$, contradicting (B.1). If $\lambda_{c} \geq 0$ and case 3) holds, then applying the definition of maximal average inter-jump time, we observe that $\lambda_{c} T+\lambda_{d} J \leq\left(\lambda_{d}+\lambda_{c} \tau\right) J+\tau N_{0} \lambda_{c} \leq$ $\lambda_{c} \tau N_{0}$. Substituting this inequality in (B.2) we find $V\left(\varphi_{q}\left(t_{0}+T, J\right)\right) \leq \bar{k} V\left(\varphi_{q}\left(t_{0}, 0\right)\right)<v_{L}$, contradicting (B.1). A contradiction has been obtained in all three cases, proving that $\bar{k} V\left(\varphi_{q}\left(t_{0}, 0\right)\right)<v_{L}$ implies $\varphi_{q}(t, j) \in V_{L}$ for all $(t, j) \in \operatorname{dom} \varphi_{q}$. Hence, $V\left(\varphi_{q}\left(t_{0}, 0\right)\right) \leq \frac{v_{L}}{\bar{k}}$ implies that, for all $\left(t_{0}+t, j\right) \in$ $\operatorname{dom} \varphi_{q}, V\left(\varphi_{q}\left(t_{0}+t, j\right)\right) \leq e^{\lambda_{c} t+\lambda_{d} j} V\left(\varphi_{q}\left(t_{0}, 0\right)\right)$.

Assumption 2 states that all trajectories of (1) are unbounded in $t$-direction, which implies $G(D) \subseteq C \cup D$. Hence, we find $\varphi_{q}\left(t_{0}+t, j\right) \in C_{e} \cup D_{e}$ for all $\left(t_{0}+t, j\right) \in \operatorname{dom} \varphi_{q}$, and we can use (8). Consequently, $d\left(\varphi_{q}\left(t_{0}+t, j\right)\right) \leq \alpha_{1}^{-1}\left(e^{\lambda_{c} t+\lambda_{d} j} \alpha_{2}\left(d\left(\varphi_{q}\left(t_{0}, 0\right)\right)\right)\right)$. With the inequalities for $\lambda_{c} t+\lambda_{d} j$ derived above, we conclude that in any of the three cases of the theorem, $d\left(\varphi_{q}\left(t_{0}+t, j\right)\right) \leq \alpha_{1}^{-1}\left(\bar{k} \alpha_{2}\left(d\left(\varphi_{q}\left(t_{0}, 0\right)\right)\right)\right)$, proving sta- 


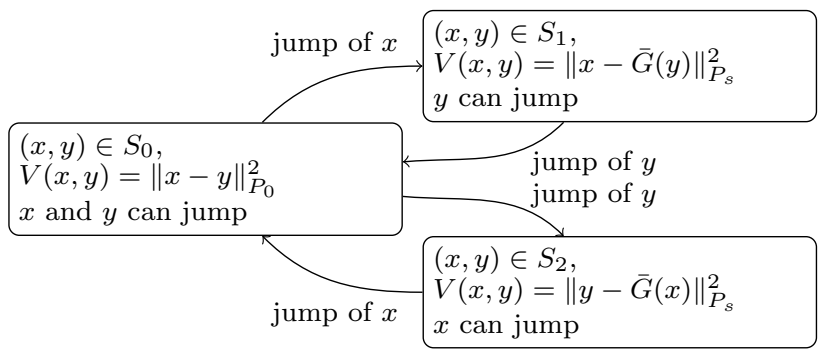

Figure B.1. The three nodes indicate when $x$ and $y$ may jump provided $V(x, y) \leq v_{L}$, with $v_{L}$ sufficiently small. When the conditions of Lemma 3 hold and, in addition, $V(x, y) \leq \max \left(1, e^{-\lambda d}\right) v_{L}$ right before a jump, then this jump satisfies the scenarios depicted by arrows.

bility with respect to $d$. Again using the mentioned inequalities, we observe that $\lambda_{c} t+\lambda_{d} j \rightarrow-\infty$ along the solutions (this limit can be used since all trajectories are unbounded in $t$-direction, cf. Assumption 2), such that $d\left(\varphi_{q}\left(t_{0}+t, j\right)\right) \rightarrow 0$. This proves asymptotic stability. When (9) and (10) hold for all $y$ such that $\left(\varphi_{x}(t, j), y\right) \in C_{e} \cup D_{e}$, then the upper bounds on $d\left(\varphi_{q}\left(t_{0}+t, j\right)\right)$ prove global asymptotic stability.

The proof of Lemma 3 employs Lemmas 3 and 7 in [5], which hinge on the observation in [5] that the set $V_{L}$ can be partitioned in three separated sets $S_{0}, S_{1}, S_{2}$ where the minimiser of $(13)$ is $\|x-y\|_{P_{0}}^{2},\|x-\bar{G}(y)\|_{P_{s}}^{2}$ or $\|\bar{G}(x)-y\|_{P_{s}}^{2}$, respectively, and, in addition, the jumps of the system (5) are restricted to the scenarios in Fig. B.1. Hence, Lemma 3 is proven by checking (9) along the scenarios in Figure B.1.

PROOF. [Proof of Lemma 3] To prove the lemma, first, we observe that [5, Lemma 7] directly guarantees that there exist functions $\alpha_{1}, \alpha_{2}$ satisfying (8). In addition, [5, Lemma 3] directly proves that there exists a sufficiently small $v_{L}>0$ such that $V$ is smooth in an open domain containing $V_{L}$. It remains to be proven that (14)-(15) imply (9).

Jumps of (11) may trigger jumps between the sets $S_{0}, S_{1}$ and $S_{2}$. From item 2) in [5, Lemma 3], we observe that for $(x, y) \in S_{1} \cap V_{L}$ and $(x, y) \in S_{2} \cap V_{L}$ jumps of $x$ and $y$, respectively, are not feasible. Consequently, when $(x, y) \in S_{0}$, both $x$ and $y$ can jump, while from $(x, y) \in$ $S_{1}$, only a jump of $y$ is feasible, and $(x, y) \in S_{2}$ implies $x \notin D$. We will now prove that (9) holds along these four jumps:

a) We first study the jump $(x, y) \rightarrow(G(x), y)$, with $(x, y) \in S_{0}$. Since 3$)$ of [5, Lemma 3] implies that $\bar{G}(y)=(L+M J) y+H+M K$ as $z_{1} y+z_{2} \leq 0$ and $x \in D$ implies $\bar{G}(x)=G(x)=(L+M J) x+H+M K$, we observe that $V(G(x), y) \leq\|G(x)-\bar{G}(y)\|_{P_{s}}^{2}=$ $\|\bar{G}(x)-\bar{G}(y)\|_{P_{s}}^{2}=(x-y)^{T}(L+M J)^{T} P_{s}(L+$ $M J)(x-y)$, such that (14) implies that (9) holds. b) For a jump $(x, y) \rightarrow(x, G(y))$ with $(x, y) \in S_{1}$, we observe $V(x, G(y)) \leq\|x-G(y)\|_{P_{0}}^{2}=\|x-\bar{G}(y)\|_{P_{0}}^{2}$, as $y \in D$. Hence, (15) implies (9) in this case.

c) For a jump $(x, y) \rightarrow(x, G(y))$, with $(x, y) \in S_{0},(9)$ directly follows from combining a) with the symmetry relation $V(x, y)=V(y, x)$.

d) For a jump $(x, y) \rightarrow(G(x), y)$ with $(x, y) \in S_{2}$, symmetry of $V$ and b) imply (9).

Hence, we have proven that (9) holds over all feasible jumps, therewith concluding the proof of the lemma.

PROOF. [Proof of Theorem 4] We prove this theorem by application of Theorem 2 . Lemma 3 proves that (8) and (9) hold for some $v_{L}>0$. Hence, we now show that the assumptions in the theorem prove that (10) is satisfied in the sub-level set $V_{L}=V^{-1}\left(\left[0, v_{L}\right]\right)$.

According to Lemma $3, V$ is differentiable in $V_{L}$, such that we evaluate $\left\langle\left.\frac{\partial V}{\partial q}\right|_{q}, f\right\rangle$ for $f \in \bar{F}_{e}\left(t, x_{d}(t, j), y\right)$ only when $q=\left(x_{d}(t, j), y\right) \in V_{L} \cap C_{e}$, where, for almost all $t, \bar{F}_{e}$ is single-valued, and we distinguish the three cases given by the minimisers of (13). If $\left(x_{d}(t, j), y\right) \in S_{0} \cap V_{L}$, then

$$
\frac{\partial V}{\partial q}=2\left(x_{d}(t, j)-y\right)^{T} P_{0}\left(I_{n}-I_{n}\right)
$$

and

$$
\bar{F}_{e}=\left(\begin{array}{c}
A x_{d}(t, j)+E+B u_{\mathrm{ff}}(t) \\
A y+E+B\left(u_{\mathrm{ff}}(t)-c_{0}\left(x_{d}(t, j)-y\right)\right)
\end{array}\right),
$$

such that (10) is guaranteed by (19).

If $\left(x_{d}(t, j), y\right) \in S_{1} \cap V_{L}$, then 3) of [5, Lemma 3] implies $\bar{G}(y)=(L+M J) y+H+M K$. Consequently

$$
\frac{\partial V}{\partial q}=2 s_{1}^{T} P_{s}\left(I_{n}-(L+M J)\right)
$$

and

$$
\bar{F}_{e}\left(t, x_{d}(t, j), y\right)=\left(\begin{array}{c}
A x_{d}(t, j)+E+B u_{\mathrm{ff}}(t) \\
A y+E+B\left(u_{\mathrm{ff}}(t)-\frac{\beta_{2}^{T} \beta_{1}(t)}{\beta_{2}^{T} \beta_{2}}-c_{1} s_{1}\right)
\end{array}\right)
$$

with $s_{1}=x_{d}(t, j)-\bar{G}(y)$ holds. Hence, we obtain $\frac{\partial V}{\partial q} \bar{F}_{e}(t, x, y)=2 s_{1}^{T} P_{s}\left(A x_{d}(t, j)+E+B u_{\mathrm{ff}}(t)-(L+\right.$ $M J) A y-(L+M J) E-(L+M J) B u_{\mathrm{ff}(t)}-\frac{\beta_{2} \beta_{2}^{T}}{\beta_{2}^{T} \beta_{2}} \beta_{1}(t)-$ $(L+M J) B c_{1} s_{1}$. With (18), we find $\frac{\beta_{2} \beta_{2}^{T}}{\beta_{2}^{T} \beta_{2}} \beta_{1}(t)=\beta_{1}(t)$, such that

$$
\begin{aligned}
& \frac{\partial V}{\partial q} \bar{F}_{e}\left(t, x_{d}(t, j), y\right)= \\
& 2 s_{1}^{T} P_{s}\left(A x_{d}(t, j)+(I-L-M J)\left(E+B u_{\mathrm{ff}}(t)\right)\right. \\
& \left.\quad-(L+M J) A y-\beta_{1}(t)+\beta_{2} c_{1} s_{1}\right) .
\end{aligned}
$$


Since $y=(L+M J)^{-1}\left(-s_{1}+x_{d}(t, j)-H-M K\right)=$ $-(L+M J)^{-1} s_{1}+G^{\circ}\left(x_{d}(t, j)\right)$, we obtain

$$
\begin{aligned}
& \frac{\partial V}{\partial q} \bar{F}_{e}\left(t, x_{d}(t, j), y\right)= \\
& \quad 2 s_{1}^{T} P_{s}\left((L+M J) A(L+M J)^{-1}+\beta_{2} c_{1}\right) s_{1},
\end{aligned}
$$

where we used the design of $\beta_{1}$. Hence, (20) guarantees that (10) holds in this case.

Now, we focus on the case $\left(x_{d}(t, j), y\right) \in S_{2} \cap V_{L}$. In that case, from 3) of [5, Lemma 3], we observe that $\max \left(0, z_{1} y+z_{2}\right)=0$ follows from $\left(x_{d}(t, j), y\right) \in S_{2} \cap V_{L}$. Hence,

$$
\frac{\partial V}{\partial q}=2 s_{2}^{T} P_{s}\left(L+M J \quad-I_{n}\right)
$$

and

$\bar{F}_{e}\left(t, x_{d}(t, j), y\right)=\left(\begin{array}{c}A x_{d}(t, j)+E+B u_{\mathrm{ff}}(t) \\ A y+E+B\left(u_{\mathrm{ff}}(t)-\frac{\beta_{4}^{T} \beta_{3}(t)}{\beta_{4}^{T} \beta_{4}}+c_{2} s_{2}\right)\end{array}\right)$

with $s_{2}=\bar{G}\left(x_{d}(t, j)\right)-y$. From (18) follows $\frac{\beta_{4} \beta_{4}^{T}}{\beta_{4}^{T} \beta_{4}} \beta_{3}(t)=$ $\beta_{3}(t)$, such that $\frac{\partial V}{\partial q} \bar{F}_{e}\left(t, x_{d}(t, j), y\right)=2 s_{2}^{T} P_{s}\left(A s_{2}-\right.$ $\beta_{3}(t)+\left(L+M J-I_{n}\right)\left(\begin{array}{c}A x_{d}(t, j)+B u_{\mathrm{ff}}(t)+E \\ A \bar{G}\left(x_{d}(t, j)\right)+B u_{\mathrm{ff}}(t)+E\end{array}\right)+$ $\left.B c_{2} s_{2}\right)$, where we used $y=\bar{G}\left(x_{d}(t, j)\right)-s_{2}$. With the design of $\beta_{3}, \beta_{4}$, we find

$$
\frac{\partial V}{\partial q} \bar{F}_{e}\left(t, x_{d}(t, j), y\right)=2 s_{2}^{T} P_{s}\left(A+\beta_{4} c_{2}\right) s_{2},
$$

such that (21) proves that (10) holds in this case. Consequently, if (19)-(21) hold, (10) is obtained. Hence, Theorem 2 proves that $x_{d}$ is asymptotically stable with respect to $d$. 